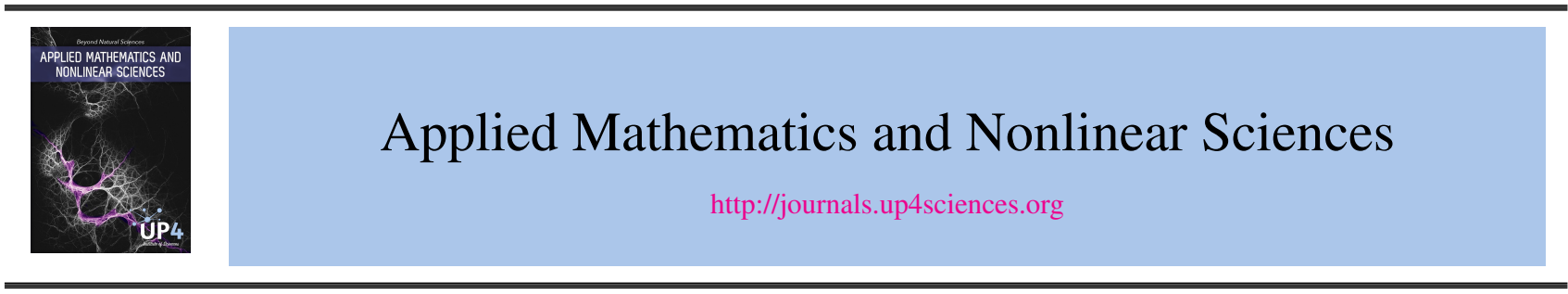

\title{
Multiplier method and exact solutions for a density dependent reaction-diffusion equation
}

\author{
M. Rosa, M. L Gandarias ${ }^{\dagger}$, \\ Departamento de Matemáticas, Universidad de Cádiz, 11500 Puerto Real, Cádiz, \\ Spain.
}

Submission Info

Communicated by Juan L.G. Guirao

Received 5th March 2016

Accepted 1st July 2016

Available online 1st July 2016

\begin{abstract}
Reaction-diffusion equations have enjoyed a considerable amount of scientific interest. The reason for the large amount of work put into studying these equations is not only their practical relevance, but also interesting phenomena that can arise from such equations. Fisher equation is commonly used in biology for population dynamics models and in bacterial growth problems as well as development and growth of solid tumours. The physical aspects of this equation are not fully understood without getting deeper into the concept of conservation laws. In [4], Anco and Bluman gave a general treatment of a direct conservation law method for partial differential equations expressed in a standard Cauchy-Kovaleskaya form. In this work we study the well known density dependent diffusion-reaction equation. We derive conservation laws by using the direct method of the multipliers.
\end{abstract}

Keywords: Lie symmetries; Partial differential equations; Conservation laws; Fisher equation

AMS 2010 codes: 76M60, 92D25, 35Q91

\section{Introduction}

Partial differential equations arise in situations in which an unknown function depends on more than one variable. Many applications of mathematics in the physical and biological sciences involve partial differential equations, and there is a great variety of techniques for obtaining information about the behavior of solutions from a population that may depend continuously on both time and location in space. The Fisher-Kolmogorov equation was proposed for populations dynamics in 1930. It shows spread of an advantageous gene in a population. The existence of solutions and traveling waves was demonstrated in 1937 [12]. The analysis and

$\dagger$ Corresponding author.

Email address: marialuz.gandarias@uca.es

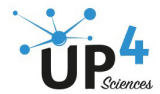


study of the Fisher equation is used to model heat and reaction-diffusion problems applied to mathematical biology, physics, astrophysics, chemistry, genetics, bacterial growth problems, development and growth of solid tumours $[5,16,20,23]$.

As described by Britton [7], generalizations of this equation are needed to more accutarely model complex diffusion and reaction effects found in many biological systems. The equation analyzed in this paper is a generalized Fisher equation in which $g(u)$ is the diffusion coefficient depending on the variable $u$, being $x$ and $t$ the independent variables, and $f(u)$ an arbitrary function

$$
u_{t}=f(u)+\left(g(u) u_{x}\right)_{x} .
$$

Equation (1) is also known as the density dependent reaction-diffusion equation which is mentioned by J.D. Murray in [19] to model the advance of an advantageous gene through a geographic region. Reaction term or source term, in which concentration is generated or degenerated by local interaction, diffusion term which causes the substances to spread out in space. Reaction-diffusion equations arise from modelling densities of particles such as substances and organisms which disperse through space as a result of the irregular movement of every particle. For some special wave speeds the equation is shown to be of Painlevé type and the general solution for these wave speeds were found in [1].

Lie symmetry analysis of differential equations provides a powerful and fundamental framework to the exploitation of systematic procedures leading to the integration by quadrature of ordinary differential equations, to the determination of invariant solutions of initial and boundary value problems and to the derivation of conservation laws [2].

In some particular cases this equation has been studied by other authors.

In the particular case of $g(u)=1$ [9], symmetry reductions and exact solutions were obtained using classical and nonclassical symmetries. An exact solution of a quasilinear Fisher equation in cylindrical coordinates

$$
u_{t}=u(1-u)+\frac{1}{x}\left[x u u_{x}\right]_{x},
$$

by using Lie classical reductions, was derived in term of the Bessel functions [6].

A complete classification of the classical symmetries and exact solutions of

$$
u_{t}=f(u)+\frac{1}{x}\left(x g(u) u_{x}\right)_{x}
$$

was obtained [22]. All the reductions were derived from the optimal system of subalgebras. Some of the reduced equations admit Lie symmetries which yield to further reductions. In [8] the form-preserving transformations were applied to derive the complete Lie symmetry classification of Eq.:

$$
u_{t}=\left(A(u) u_{x}\right)_{x}+B(u) u_{x}+C(u)
$$

with $B(u)=0$, Eq. (4) becomes the so called density dependent equation. In [10], Eq. (4) have studied from the point of view of conservation laws.

Owing to the great advance in computation in the last few years, a great progress has being made in the development of methods and their applications for finding solitary traveling-wave solutions of nonlinear evolution equations. Many solutions of nonlinear partial differential equations have been found by one or other of these methods. In [26], Wang introduced a method which is called the $\frac{G^{\prime}}{G}$-expansion method to look for traveling wave solutions of nonlinear evolution equations. The main ideas of the proposed method are that the traveling wave solutions of a nonlinear evolution equation can be expressed by a polynomial in $\frac{G^{\prime}}{G}$, where $G=G(z)$ satisfies the linear second order ordinary differential equation

$$
G^{\prime \prime}(z)+\omega G^{\prime}(z)+\zeta G(z)=0 .
$$


It is known that conservation laws play a significant role in the solution process of an equation or a system of differential equations. Although not all of the conservation laws of partial differential equations (PDEs) may have physical interpretation they are essential in studying the integrability of the PDEs.

For variational problems, the Noether theorem can be used for the derivation of conservation laws. For non variational problems there are different methods for the construction of conservation laws. In [4], Anco and Bluman gave a general algorithmic method to find all conservations laws for evolution equations like Eq. (1). Many recent papers using this method have been published in [3,13,14] In a previous paper [15], for equation (1) we have determined the subclasses of equations which are nonlinearly self-adjoint. And we have determined, by using the Lie generators of (1) and the notation and techniques introduced by Ibragimov [17], some nontrivial conservation laws. After Ibragimov's results several papers appeared concerned with self-adjointness and its applications to PDEs [24,25]. In this work, we derive conservation laws by using the direct method of the multipliers [4]. Furthermore, due to the fact that equation (1) admits groups of space and time traslations, we look for travelling wave solutions of the density dependent reaction-diffusion equation (1) with physical interest. In order to do that we apply the well known $\frac{G^{\prime}}{G}$-expansion method.

\section{Lie symmetries}

We start by applying the classical Lie group method to equation (1). Most of the required theory and description of the method can be found in Olver [21]. To apply the classical method to equation (1), one looks for infinitesimal generators of the form

$$
V=\xi(x, t, u) \partial_{x}+\eta(x, t, u) \partial_{t}+\psi(x, t, u) \partial_{u},
$$

that leave invariant this equation. By applying these techniques to equation (1), we find that, if $f$ and $g$ are arbitrary functions, the symmetries that are admitted by (1) are

$$
\mathbf{v}_{1}=\partial_{x}, \mathbf{v}_{2}=\partial_{t}
$$

The following functional forms of $f$ and $g$ which have extra symmetries are are given in Table 1:

Table 1: Functions and generators.

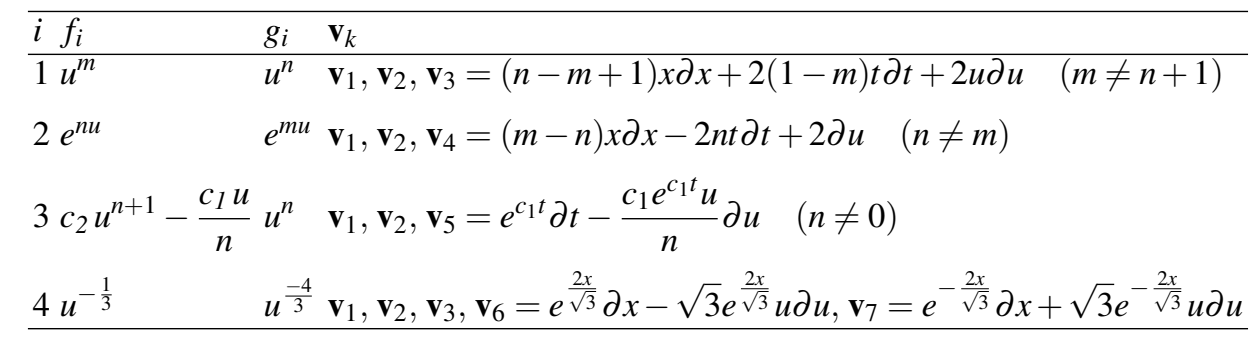

\section{Optimal systems and reductions}

In order to construct the one-dimensional optimal system, following Olver in [21], we construct the commutator tables and the adjoint tables which show the separate adjoint actions of each element in $\mathbf{v}_{i}, i=1 \ldots n$, as it acts on all other elements. This construction is done easily by summing the Lie series. The corresponding generators of the optimal system of subalgebras are:

Table 2: Optimal systems. 


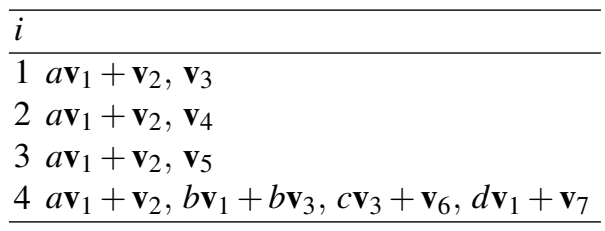

In the following, similarity solutions and reductions of the equation (1) to ordinary differential equations (ODEs) are obtained using the generators of the optimal system.

For $f(u)$ and $g(u)$ arbitrary functions with the generator $a \mathbf{v}_{1}+\mathbf{v}_{2}$, the similarity variable and similarity solution are

$$
z=x-a t, u=h(z) .
$$

Substituting into (1) we obtain the reduced ODE

$$
g h_{z z}+g_{h} h_{z}^{2}+a h_{z}+f=0,
$$

with $f=f(h)$ and $g=g(h)$ arbitrary functions.

Table 3: Similarity solutions.

\begin{tabular}{llll}
\hline$i j \mathbf{w}_{j}$ & $z_{j}$ & $u_{j}$ \\
\hline $11 \mathbf{v}_{3}$ & $t^{\frac{n-m+1}{2 m-2}} x$ & $h x^{\frac{2}{n-m+1}}$ \\
22 & $\mathbf{v}_{4}$ & $t^{\frac{n-m}{2 m}} x$ & $\frac{2 \log (x)}{n-m}+h$ \\
3 & $3 \mathbf{v}_{5}$ & $x$ & $h e^{-\frac{c_{1} t}{n}}$ \\
$44 b \mathbf{v}_{1}+\mathbf{v}_{3}$ & $\frac{8 x-3 b \log t}{8}$ & $h t^{\frac{3}{4}}$ \\
$45 c \mathbf{v}_{3}+\mathbf{v}_{6}$ & $-\frac{4 c e^{-\frac{2 x}{\sqrt{3}}}}{\sqrt{3}}-\log t h e^{-\sqrt{3} c e^{-\frac{2 x}{\sqrt{3}}}-\sqrt{3} x}$ \\
4 & $6 d \mathbf{v}_{3}+\mathbf{v}_{7} \frac{4 d e^{\frac{2 x}{\sqrt{3}}}}{\sqrt{3}}-\log t$ & $h e^{\sqrt{3} d e^{\frac{2 x}{\sqrt{3}}}+\sqrt{3} x}$ \\
\hline
\end{tabular}

Table 4: Reduced ODEs:

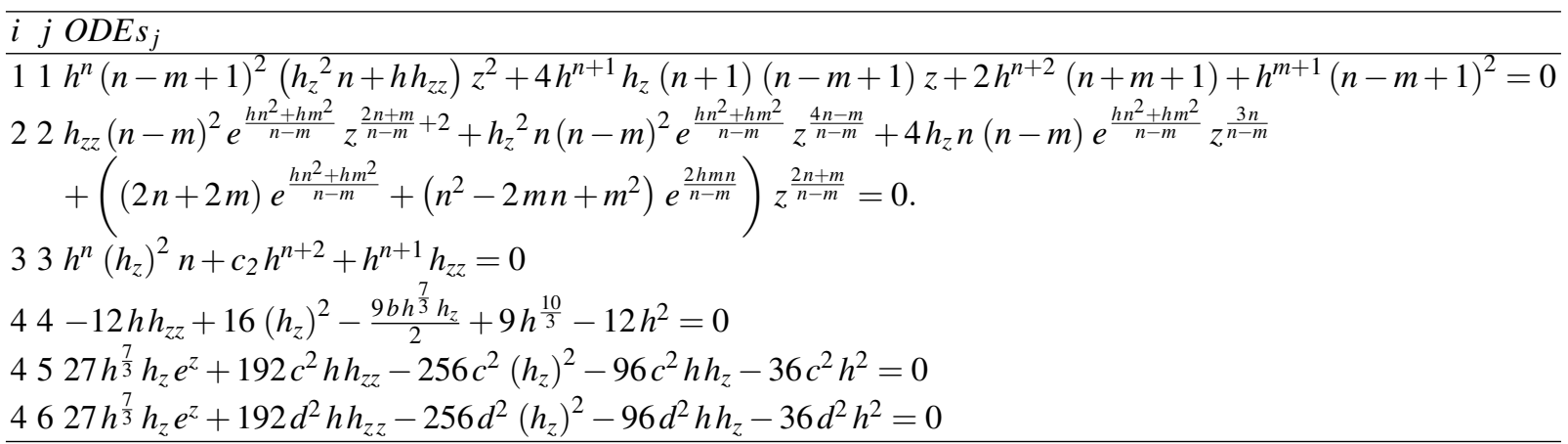

\section{Travelling wave solutions}

From generators $\mathbf{v}_{1}$ and $\mathbf{v}_{2}$ we can obtain traveling wave solutions for Eq. (1). By substituting the similarity variable and the similarity solution

$$
z=x-\lambda t, \quad u(x, t)=h(z) .
$$

into

$$
u_{t}=f(u)+\left(g(u) u_{x}\right)_{x}
$$


we obtain

$$
-h_{z} \lambda-g h_{z z}-g_{h}\left(h_{z}\right)^{2}-f=0 .
$$

To apply the $\frac{G^{\prime}}{G}$-expansion method to Eq. (8) we suppose that the solutions can be expressed by a polynomial in $\frac{G^{\prime}}{G}$ in the form

$$
h=\sum_{i=0}^{n} a_{i}\left(\frac{G^{\prime}}{G}\right)^{i}
$$

where $G=G(z)$ satisfies the linear second order ODE

$$
G^{\prime \prime}(z)+\omega G^{\prime}(z)+\zeta G(z)=0,
$$

$a_{i}, i=0, \ldots, n, \alpha$ and $\beta$ are constants to be determined later, $a_{n} \neq 0$.

The general solutions of Eq. (10) are:

- If $\omega^{2}-4 \zeta>0$,

$$
\begin{aligned}
G(z)= & c_{1} \cosh \left(\frac{z \omega}{2}-\frac{1}{2} z \sqrt{\omega^{2}-4 \zeta}\right)+c_{2} \cosh \left(\frac{\omega z}{2}+\frac{1}{2} \sqrt{\omega^{2}-4 \zeta z}\right) \\
& -c_{1} \sinh \left(\frac{z \omega}{2}-\frac{1}{2} z \sqrt{\omega^{2}-4 \zeta}\right)-c_{2} \sinh \left(\frac{\omega z}{2}+\frac{1}{2} \sqrt{\omega^{2}-4 \zeta} z\right)
\end{aligned}
$$

- If $\omega^{2}-4 \zeta<0$,

$$
G(z)=\left[c_{2} \cos \left(\frac{1}{2} z \sqrt{4 \zeta-\omega^{2}}\right)+c_{1} \sin \left(\frac{1}{2} z \sqrt{4 \zeta-\omega^{2}}\right)\right]\left(\cosh \left(\frac{z \omega}{2}\right)-\sinh \left(\frac{z \omega}{2}\right)\right) .
$$

- If $\omega^{2}=4 \zeta$,

$$
G(z)=\left(c_{2}+c_{1} z\right)\left(\cosh \left(\frac{z \omega}{2}\right)-\sinh \left(\frac{z \omega}{2}\right)\right) .
$$

In order to determine the positive number $n$ in (9) we concentrate our attention on the leading terms of (8). These are the terms that lead to the least positive $p$ when substituting a monomial $h=\frac{a}{z^{p}}$ in all the items of equation, Kudryashov in [18]. The homogeneous balance between the leading terms provides us with the value of $n$. To find them we substitute $h=\frac{a}{z^{p}}$ in all the items of this equation.Setting $g(h)=h^{m}$, we compare $g(h) h^{\prime \prime}$ and $g^{\prime}(h)\left(h^{\prime}\right)^{2}: p m+p+2=p(m-1)+(p+1)^{2} \Rightarrow p=1 m:$ arbitrary.

By using (9) and (10) we obtain

$$
\begin{aligned}
h & =a_{n}\left(\frac{G^{\prime}}{G}\right)^{n}+\cdots \\
h^{2} & =a_{n}^{2}\left(\frac{G^{\prime}}{G}\right)^{2 n}+\cdots \\
h^{\prime \prime} & =n(n+1) a_{n}\left(\frac{G^{\prime}}{G}\right)^{n+2}+\cdots
\end{aligned}
$$

Considering the homogeneous balance between $h^{\prime \prime}$ and $h^{2}$ in (8), based on (15) and (16), we require that $n m+n+2=n(m-1)+(n+1)^{2} \Rightarrow n=1$, we can write (9) as

$$
h=a_{0}+a_{1}\left(\frac{G^{\prime}}{G}\right),
$$

$a_{1} \neq 0$.

From the general solutions of (10), setting without loss of generality $a_{0}=a_{1}=1$, we obtain that: 
- For

$$
g(h)=h^{m}
$$

and

$$
f(h)=\left(h^{2}-2 h+2\right)\left(\lambda-2 h^{m+1}+2 h^{m}\right)-2 h^{m-1}\left(h^{2}-2 h+2\right)^{2} m
$$

we get the solution

$$
h_{1}(z)=\frac{c_{1} \cos z-c_{2} \sin z}{c_{1} \sin z+c_{2} \cos z}+1 .
$$

The corresponding solution for the generalized Fisher equation where

$$
g(u)=u^{m}
$$

and

$$
f(u)=\left(u^{2}-2 u+2\right)\left(\lambda-2 u^{m+1}+2 u^{m}\right)-2 u^{m-1}\left(u^{2}-2 h+2\right)^{2} m
$$

is

$$
u_{1}=\frac{c_{2} \sin (t \lambda-x)+c_{1} \cos (t \lambda-x)}{c_{2} \cos (t \lambda-x)-c_{1} \sin (t \lambda-x)}+1
$$

- For

$$
g(h)=h^{m}
$$

and

$$
f(h)=(h-2) h\left(\lambda-h^{m+1} m+2 h^{m} m-2 h^{m+1}+2 h^{m}\right)
$$

we get the solution

$$
h(z)=\frac{c_{2} \sinh z+c_{1} \cosh z}{c_{1} \sinh z+c_{2} \cosh z}+1
$$

The corresponding solution for the generalized Fisher equation where

$$
f(u)=(u-2) u\left(\lambda-u^{m+1} m+2 u^{m} m-2 u^{m+1}+2 u^{m}\right)
$$

is

$$
u_{2}=\frac{c_{2} \sinh (x-\lambda t)+c_{1} \cosh (x-\lambda t)}{c_{1} \sinh (x-\lambda t)+c_{2} \cosh (x-\lambda t)}+1
$$

- For $g(h)=h^{m}$ and

$$
f(h)=h\left(h \lambda-2 h^{m+2} m-2 h^{m+2}\right)
$$

we get the solution

$$
h(z)=\frac{\left(c_{1} z+c_{2}\right)(\sinh z-\cosh z)+c_{1}(\cosh z-\sinh z)}{\left(c_{1} z+c_{2}\right)(\cosh z-\sinh z)}+.
$$

The corresponding solution for the generalized Fisher equation where $g(u)=u^{m}$ and

$$
f(u)=u\left(u \lambda-2 u^{m+2} m-2 u^{m+2}\right)
$$

is

$$
u_{3}=h(x-\lambda t) .
$$

We now search for exact solutions in terms of the Jacobi elliptic functions. For

$$
g(h)=h^{m}
$$


and

$$
f(h)=-\sqrt{1-h} \sqrt{h+1} \sqrt{1-h^{2} p}+h^{m+1}(m+1)(p+1)-h^{m+3}(m+2) p-h^{m-1} m
$$

we get the solution

$$
h(z)=\operatorname{Sn}(z \mid p) .
$$

In the particular case $p=1$ we get a kink solution $h(z)=\tanh (z)$, then, the corresponding solution for Eq. (1) is

$$
u=\tanh (x-\lambda t)
$$

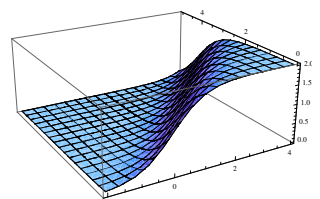

Fig. 1 Kink solution with $\lambda=1$

\section{Multiplier conservation laws method}

In [4] Anco and Bluman gave a general treatment of a direct conservation law method for partial differential equations expressed in a standard Cauchy-Kovaleskaya form in particular for evolution equations

$$
u_{t}=G\left(x, u, u_{x}, u_{x x}, \ldots, u_{n x}\right) .
$$

The nontrivial conservation laws are characterized by a multiplier $\lambda$ with no dependence on $u_{t}$ satisfying

$$
\hat{E}[u]\left(\Lambda u_{t}-\Lambda G\left(x, u, u_{x}, u_{x x}, \ldots, u_{n x}\right)\right)=0 .
$$

Here

$$
\hat{E}[u]:=\frac{\partial}{\partial u}-D_{t} \frac{\partial}{\partial u_{t}}-D_{x} \frac{\partial}{\partial u_{x}}+D_{x}^{2} \frac{\partial}{\partial u_{x x}}+\ldots
$$

is the Euler operator. The conservation law will be written

$$
D_{t}\left(\Phi^{t}\right)+\left.D_{x}\left(\Phi^{x}\right)\right|_{\Delta}=0,
$$

where $\Phi^{t}$ and $\Phi^{x}$ are called the conserved densities. The conserved current must satisfy

$$
\Lambda=\hat{E}[u] \Phi^{t}
$$

and the flux $\Phi^{x}$ is given by [11]

$$
\Phi^{x}=-D_{x}^{-1}(\Lambda G)-\frac{\partial \Phi^{t}}{\partial u_{x}} G+G D_{x}\left(\frac{\partial \Phi^{t}}{\partial u_{x x}}\right)+\ldots
$$

For Eq. (1), we can state:

Case 1: For $f_{u}-k^{2} g=0$ with $k$ constant, we get the following multipliers:

$$
\Lambda_{1}=\sin (k x), \Lambda_{2}=\cos (k x) .
$$

Case 2: For $f_{u}+k^{2} g=0$ with $k$ constant, we get the following multipliers:

$$
\Lambda_{3}=e^{k x}, \Lambda_{4}=e^{-k x} .
$$

Case 3: For $f(u)=a u+b$ with $a, b$ constants and $g(u)$ arbitrary we obtain the following multipliers:

$$
\Lambda_{5}=x e^{-a t}, \Lambda_{6}=e^{-a t} .
$$




\section{Conservation laws}

Case 1: For $f_{u}-k^{2} g=0$

Associated to the multipliers, from (21) and (22), we obtain the corresponding conserved densities and fluxes: 1.-

$$
\begin{aligned}
\Lambda_{1} & =\sin (k x), \\
\phi^{t} & =\sin (k x) u, \\
\phi^{x} & =-\sin (k x) g(u) u_{x}+\cos (k x) k \int g(u) d u .
\end{aligned}
$$

2.-

$$
\begin{aligned}
\Lambda_{2} & =\cos (k x), \\
\phi^{t} & =\cos (k x) u \\
\phi^{x} & =-\cos (k x) g(u) u_{x}-\sin (k x) k \int g(u) d u .
\end{aligned}
$$

Case 2: For $f_{u}+k^{2} g=0$

Associated to the multipliers, from (21) and (22), we obtain the corresponding conserved densities and fluxes: 1.-

$$
\begin{aligned}
\Lambda_{3} & =e^{k x} \\
\phi^{t} & =e^{k x} u, \\
\phi^{x} & =-e^{k x}\left(g(u) u_{x}-k \int g(u) d u\right) .
\end{aligned}
$$

2.-

$$
\begin{aligned}
\Lambda_{4} & =e^{-k x}, \\
\phi^{t} & =e^{-k x} u, \\
\phi^{x} & =-e^{-k x}\left(g(u) u_{x}+k \int g(u) d u\right) .
\end{aligned}
$$

Case 3: For $f=a u+b$ and $g$ arbitrary. Associated to the multipliers, from (21) and (22), we obtain the corresponding conserved densities and fluxes:

1.-

$$
\begin{aligned}
\Lambda_{5} & =e^{-a t}, \\
\phi^{t} & =\frac{\mathrm{e}^{-a t}(a u+b)}{a}, \\
\phi^{x} & =-\mathrm{e}^{-a t} g(u) u_{x} .
\end{aligned}
$$

2.-

$$
\begin{aligned}
\Lambda_{6} & =x e^{-a t}, \\
\phi^{t} & =\frac{x \mathrm{e}^{-a t}(a u+b)}{a}, \\
\phi^{x} & =\mathrm{e}^{-a t}\left(-x g(u) u_{x}+\int g(u) d u\right) .
\end{aligned}
$$




\section{Conclusions}

In this paper, we have obtained Lie symmetries of the density dependent reaction-diffusion equation (1) and we have calculated the optimal system of one-dimensional subalgebras of the invariant equation. By using the classical Lie group method, we obtained reductions to ODE's and some exact solutions. In order to obtain travelling-wave solutions of the generalized Fisher equation (1) we have employed the $\frac{G^{\prime}}{G}$-expansion method. We have obtained some exact solutions among them a kink solution. By the multipliers method we have obtained some nontrivial conservation laws via integral formulae.

\section{References}

[1] Ablowitz, M.J., Zeppetella, A. (1979), Explicit solutions of Fisher's equation for a special wave speed. Bull. Math. Biol, 41: 835-840.

[2] de la Rosa, R. and Bruzón, M.S. (2016), On the classical and nonclassical symmetries of a generalized Gardner equation. Applied Mathematics and Nonlinear Sciences, 1(1): 263?272. doi 10.21042/AMNS.2016.1.00021

[3] Adem, K.R. and Khalique, C.M. (2015), Symmetry Analysis and Conservation Laws of a Generalized TwoDimensional Nonlinear KP-MEW Equation. Mathematical Problems in Engineering, Volume 2015, Article ID 805763, doi 10.1155/2015/805763

[4] Anco, S.C., Bluman G. (2002), Direct construction method for conservation laws for partial differential equations Part II: General treatment Euro. Jnl of Applied mathematics, 41: 567-585. doi 10.1017/S095679250100465X

[5] Belmonte-Beitia, J., Calvo, G.F., Pérez-García, V.M. (2014), Effective particle methods for the Fisher-Kolmogorov equations: Theory and applications to brain tumor dynamics. Communications in Nonlinear Science and Numerical Simulation 19: 3267-3283 . doi 10.1016/j.cnsns.2014.02.004

[6] Bokhari, A.H., Mustafà M.T., Zaman F.D. (2008) An exact solution of a quasilinear Fisher equation in cylindrical coordinates. Nonlinear Analysis 69: 4803-4805. doi 10.1016/j.na.2007.11.012

[7] Britton, N.F. (1989), Aggregation and the competitive exclusion principle. J. Theor. Biol. 136: 57-66.

[8] Cherniha, R., Serov, M., Rassokha, I. (208), Lie symmetries and form-preserving transformations of reaction-diffusionconvection equations, J.Math. Anal. Appl. 342: 136-1379.

[9] Clarkson, P. A., Mansfield E. L. (1993) Symmetry reductions and exact solutions of a class of nonlinear heat equations. Physica D 70: 250-288.

[10] Bruzón, M.S., Gandarias, M.L., de la Rosa, R. (2014) Conservation laws of a family of reaction- diffusion-convection equations, Localized Excitations in Nonlinear Complex Systems, Series: Nonlinear Systems and Complexity 7: 403417 ISBN: 978-3-319- 02057-0

[11] Euler, N. and Euler, M. (2009), On nonlocal symmetries, nonlocal conservation laws and nonlocal transformations of evolution equations: two linearisable hierachies, Journal of Nonlinear Mathematical Physics 6: 489-504.

[12] Fisher, R.A. (1937), The wave of advance of advantageous genes. Ann.Eugenics 7: 353-69.

[13] Gandarias, M.L. and Khalique, C.M. (2016), Symmetries solutions and conservation laws of a class of nonlinear dispersive wave equations. Communications in Nonlinear Science and Numerical Simulation 32: 114-121. doi 10.1016/j.cnsns.2015.07.010

[14] Gandarias, M.L., Bruzón, M.S., and Rosa M. (2015), Symmetries and Conservation Laws for Some Compacton Equation. Mathematical Problems in Engineering, Volume 2015, Article ID 430823, doi 10.1155/2015/430823

[15] Gandarias, M.L., Bruzón, M.S., Rosa M. (2013), Nonlinear self-adjointness and conservation laws for a generalized Fisher equation. Commun. Nonlinear Sci. Numer. Simulat. 18: 1600-1606. doi 10.1016/j.cnsns.2012.11.023

[16] Fitzhugh, R. (1961), Impulses and physiological states in theoretical models of nerve membrane. Biophys J 1: 445-66.

[17] Ibragimov, N. H. (2007), A new conservation theorem J. Math. Anal. Appl. 333: 311-28. doi 10.1016/j.jmaa.2006.10.078

[18] Kudryashov, N.A. (2009), On "new travelling wave solutions" of the KdV and the KdV-Burgers equations. Commun. Nonl. Sci. Numer. Simulat, 14 1891-1900.

[19] Murray, J.D. (2002), Mathematical Biology. Third Edition. Springer-Verlag New York Berlin Heidelberg.

[20] Nagumo, J.S., Arimoto, S., Yoshizawa, S. (1962), An active pulse trasmission line simulating nerve axon Proc IRE 50: 2061-71.

[21] Olver, P.J.(1986), Applications of Lie Groups to Differential Equations. Springer, Berlin.

[22] Rosa, M., Bruzon, M.S., Gandarias, M.L. (2015) Symmetry analysis and exact solutions for a generalized Fisher equation in cylindrical coordinates. Commun Nonlinear Sci Numer Simulat, 25, 74-83. doi 10.1016/j.cnsns.2015.01.010

[23] Swanson, K. R., Bridgea, C., Murray, J.D., Ellsworth, C., Alvord, Jr. (2003), Virtual and real brain tumors: using mathematical modeling to quantify glioma growth and invasion. Journal of the Neurological Sciences, 216: 1-10. 
[24] Tracinà, R., Bruzón. M. S. and Gandarias, M. L. (2016), On the nonlinear self-adjointness of a class of fourth-order evolution equations. Applied Mathematics and Computation 275: 299-304. doi 10.1016/j.amc.2015.11.079

[25] Tracinà, R., Freire, I. L. and Torrisi, M. (2016), Nonlinear self-adjointness of a class of third order nonlinear dispersive equations. Communications in Nonlinear Science and Numerical Simulation, 32: 225-233. doi 10.1016/j.cnsns.2015.08.016

[26] Wang, N., Li, X. and Zhang, J. (2008), The $\frac{G^{\prime}}{G}$-expansion method and travelling wave solutions of nonlinear evolution equations in mathematical physics. Phys. Lett. A 372: 417.

OUP4 Sciences. All rights reserved. 